\title{
Hepatitis B vaccine contract goes to untested product
}

\section{London}

SmithKLINE Biologicals (SKB) and Merck Sharp and Dohme (MSD), two producers of the world's first genetically engineered yeast-derived hepatitis B vaccine which is billed as cheaper and more easily available than the old plasma-derived vaccine, have lost a major international supply contract for the vaccine to a small but growing South Korean pharmaceutical company.

The Korea Green Cross (no relation to its Japanese namesake) was last month awarded a contract to supply 425,500 doses of its locally manufactured plasmaderived Hepavax B vaccine, over five years, to Indonesian infants, at $\$ 0.95$ a dose, in the first of a series of pilot vaccination projects planned for South-East Asia, Africa and Latin America.

The tender was a brainchild of the USbased International Task Force on hepatitis B, a loose affiliation of nine of the world's leading hepatitis experts that operates through an independent Seattlebased charity. At a recent meeting in London, Professor Palmer Beasley, a task-force member, pointed out that "with the AIDS pandemic, WHO [World Health Organization] is not in a position to address the rising incidence in the numbers of hepatitis B carriers and cases".

Frustrated by the lack of progress in

\section{Why the world needs population biology to solve its problems}

\section{Washington}

CONSERVATIONISTS are sharpening their arguments. During the past month, pressure from Washington-based groups has led the World Bank to withdraw support from African cattle-ranching projects that would cause long-term economic harm and the Agency for International Development to publish lists of its own projects that stress short-term economic benefit at the expense of the environment. Now it is the turn of the Club of Earth, a pressure group of senior academic ecologists, to issue a statement stressing that support for population biology makes economic sense.

The club, whose members are drawn from the National Academy of Sciences and include G. Evelyn Hutchinson and E.O. Wilson, point out that it is population biology that solves "practical questions about maximum sustainable yields for fisheries, control of agricultural pests, containment of epidemics..." and that basic research is needed to keep the environment in shape just as much "as basic research in molecular biology is needed for treating human ills".

There the comparison ends. Financial support for biomedical research in the United States is more than two orders of magnitude higher than support for population biology. The major funding agency for population biology (taken to include ecology, ecosystem studies and systematic biology) is the National Science Foundation, which spends approximately $\$ 60$ million a year. It has "recently been increasing funding faster than for molecular biology" but still not fast enough to satisfy many ecologists. Jared Diamond, a Club of Earth member from the University of California at Los Angeles, points out that most ecology graduate students have to leave the field and that any job opening will at tract around 300 applicants.

Better knowledge of population biology could have helped avoid the collapse of the Peruvian fishery and the Californian sardine fishery, according to the Club of Earth. The former alone lost the world fishmeal worth $\$ 1,000$ million a year.

The evolution of malarial resistance, the seriousness of the AIDS (acquired immune deficiency syndrome) epidemic and the likely impact of genetically engineered organisms on the environment are all areas where failure to support population biology is preventing serious answers from emerging. Lack of taxonomists prevents the cataloguing of potentially valuable species, particularly in the tropics.

Diamond points out that as much as one-third of tropical biomass circulates through ants and termites but there are perhaps only six people in the world capable of classifying them. Cornell University's Tom Eisner, another club member, believes that failure to research the chemical interactions of plants, animals and fungi is slowing the development of more intelligent methods of pest control and will, in the long run, harm agriculture.

The kind of new perspective the club wants will not be easy to achieve and is unlikely to appear just by tinkering with research grants. Rather, there has to be an acceleration in the changes that are now occurring in political organizations such as the World Bank alongside a wider recognition of what population biology could contribute. To achieve that will require skilful management of Washington's complex political ecosystem - a fair intellectual challenge for the club's academics. Alun Anderson curbing the spread of the virus despite the availability of a vaccine since 1981, these experts, led now by Dr James Maynard, late of the US Centers for Disease Control, have sought to force down the cost of the vaccine to around $\$ 1$ a dose, to help developing countries to provide mass public-sector immunization programmes.

Price has certainly been a major stumbling block in many parts of Asia and Africa where one in ten are carriers of the hepatitis $\mathrm{B}$ virus. Of these, about half (2 million) will die from liver cirrhosis and liver cancer. China, Taiwan and South Korea all produce plasma-derived vaccines locally to meet domestic demand. None of these vaccines has been tested for safety and efficacy by an independent regulatory authority.

Health professionals now worry that in their zeal to get the vaccine to countries that need it, at a price they can afford, the task force may be endorsing a potentially substandard product. In evaluating the different bids, Richard Mahoney, executive secretary of the task force, said: "We did not wish to act as a regulatory agency and we did not question manufacturers' data on vaccine safety and efficacy". The task force also took the unorthodox step of declaring all vaccines acceptable if approved by the national regulatory authority of the country of origin. A spokesman for WHO confirmed that the Korean vaccine Hepavax B does not yet have the WHO seal of approval.

Although the Korea Green Cross has sold more than ten million doses of its vaccine in South Korea in the past five years, other vaccine manufacturers are worried that the company had produced only, two batches of the special paediatric dose designed to meet the tender specification. Western regulatory authorities demand the manufacture of a minimum of five consecutive batches to establish vaccine stability before product approval can be granted.

Such is the demand for a cheap hepatitis vaccine in many parts of South-East Asia that some countries are willing to take a gamble rather than wait for the major companies to lower their price. Although prices of the new genetically engineered vaccine, at $\$ 15$ per dose, are half the price of the older plasma-derived type, they are still too high to make mass vaccination programmes feasible. It is hoped, however, that high-volume orders will lower the cost of the recombinant vaccine still further.

Belgium-based SmithKline Biologicals, whose rDNA hepatitis vaccine, Engerix $B$, was licensed in the United Kingdom this week, "pledges a commitment to the philosophy of low-cost hepatitis vaccines, provided that the volume of orders is sufficiently high", states Engerix B project manager Robert Hackett.

Miriam Ryan 